\title{
The death line of millisecond pulsars
}

\author{
Claes-Ingvar Björnsson \\ Stockholm Observatory, S-133 36 Saltsjöbaden, Sweden
}

\begin{abstract}
The distribution of millisecond pulsars, just as normal pulsars, is affected by a death line. Since a large fraction of millisecond pulsars lie close to the derived death line, a significant number of neutron stars may exist which are not observed as millisecond pulsars. This makes the birth rate discrepancy between millisecond pulsars and their assumed low mass $\mathrm{x}$-ray binary progenitors more acute.
\end{abstract}

\section{Polar cap dynamics}

When particles can flow freely from the neutron star surface, the main contribution to the potential, within one scale height of the magnetic field (B) above the surface, is due to the dragging of inertial frames (Muslimow \& Tsygan 1992). The potential drop is close to the maximum one found by Ruderman \& Sutherland (1975), except that its scale height $\left(z_{o}\right)$ is the stellar radius (R) instead of the polar cap width $\left(r_{p} \simeq 10^{4} P^{-1 / 2}\right)$. The resulting pulsar death lines are very similar in the two cases.

\section{Synchrotron versus curvature radiation}

The primary electrons (positrons) accelerated in the polar gap induce a $e^{+} / e^{-}$ cascade. The $e^{+} / e^{-}$pairs are produced with a finite momentum perpendicular to the magnetic field direction. For low magnetic field and high electric field in the gap, the positrons (electrons) returning to the star do not have time to radiate away this momentum before reaching the stellar surface. The Lorentz factor of the perpendicular momentum $\left(\Gamma_{p}\right)$ in the gap is typically

$$
\Gamma_{p} \sim 10\left(\frac{R_{6}^{2} R}{B_{9} P_{-2}^{2} z_{o}}\right)^{1 / 2}
$$

This opens the possibility that synchrotron radiation can produce $e^{+} / e^{-}$pairs more efficiently than curvature radiation; hence, a new and less restrictive death line would result. Figure 1 shows the death line including synchrotron radiation (Björnsson 1996). It is seen that for millisecond pulsars, the death line is due to synchrotron radiation (This conclusion does not depend on whether the polar gap breaks down or not.). The transition between curvature and synchrotron radiation occurs for $B \sim 10^{9}-10^{1} 0 \mathrm{G}$ and is due to radiative reaction. The slopes of the two death lines are rather similar but the synchrotron one is shifted 


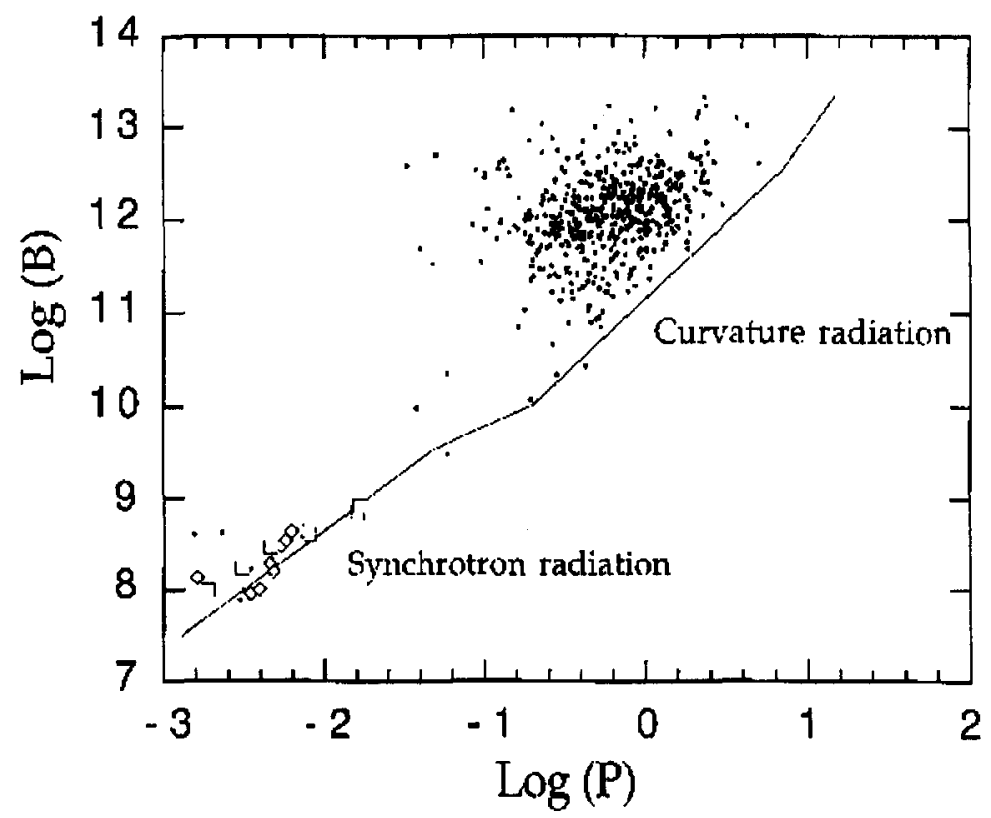

Figure 1. The calculated pulsar death line as compared to the observed distribution of $\mathrm{B}$ - and $\mathrm{P}$-values for pulsars not belonging to globular clusters (courtesy F. Camilo). The squares denote pulsars with unpublished corrections (F. Camilo, private communication) and diamonds those pulsars whose $\dot{\mathrm{P}}$ 's have been corrected for kinematic contributions.

(for a given B-value), as compared to an extrapolation of the standard curvature one, to shorter periods by a factor $\left(\alpha B_{q} / B\right)^{2 / 13} \sim 3$, where $\alpha \approx 1 / 137$ and $B_{q}=4.4110^{13} \mathrm{G}$. Although the absolute position of the death lines are somewhat uncertain, the magnitude of this shift is quite insensitive to the particular model used for the polar gap/magnetosphere; it depends mainly on the different emission properties of the two radiation mechanisms.

The main things to notice from Figure 1 are: (i) The predicted shift between normal and millisecond pulsars is supported by the observations, which implies that also millisecond pulsars are affected by a death line. (ii) The majority of millisecond pulsars lie close to the death line, suggesting a substantial number of neutron stars on the no-pulsar side.

\section{References}

Björnsson, C.-I. 1996, ApJ, In Press

Muslimow, A.G., \& Tsygan, A.I. 1992, MNRAS, 255, 61

Ruderman, M.A., \& Sutherland, P.G. 1975, ApJ, 196,51 